\title{
From Sméagol to Gollum: Mechanical Stress and Bone Remodelling
}

\author{
Nicole Pace \\ University of Malta, Gozo, Malta \\ Email: nicole.pace.14@um.edu.mt
}

How to cite this paper: Pace, N. (2018) From Sméagol to Gollum: Mechanical Stress and Bone Remodelling. Open Journal of Orthopedics, 8, 148-167. https://doi.org/10.4236/ojo.2018.84018

Received: November 12, 2017

Accepted: April 17, 2018

Published: April 20, 2018

Copyright $\odot 2018$ by author and Scientific Research Publishing Inc. This work is licensed under the Creative Commons Attribution-NonCommercial International License (CC BY-NC 4.0). http://creativecommons.org/licenses/by-nc/4.0/ (c) (i) \&) Open Access

\begin{abstract}
This imaginary transformation from Sméagol to Gollum is a dramatization of the illusive repercussions of mechanical stress affecting bone. This paper presents the main ideas of mechanical stress and bone remodelling from a novel's perspective. The object of this study is to provide evidence for new ways to explore bone's functional adaptation to mechanical stress made through the copious interpretation and integration of new and existing literature. It tackles the underlying biology of bone cells and how they detect and react to strain stimuli. The different types of mechanical demands in daily activities are sifted through and any misconceptions found fallible in literature are refined. A personal experience of a stress fracture is reviewed to parallel the implications that lead to the incident with the findings on the link between mechanical stress and bone remodelling. Some factors regarding age, gender and ethnicity and the interplay with mechanical stress influencing bone remodelling are considered. Brief overviews of three new medical novelties in bone healing are outlined, hoping that these interventions of proper medical techniques can be a change for the better: one from Gollum to Sméagol rather than vice versa.
\end{abstract}

\section{Keywords}

Mechanical Stress, Bone Remodelling Cycle, Mechanostat Model, Stress Fracture

\section{Introduction}

In the movie adaptation of the great work of fiction that is "The Lord of the Rings", movie director, Peter Jackson presents us with the lengthy and multi-faceted transformation that led the weak hobbit Sméagol to turn into the quasi-immortal Gollum. In this imaginary change, bones must have been broken and reformed tens of times at the least, over its nine-hundred-year period. Thus 
in a few minutes, we witness enormous variation in the creature's overall structure; facial features; garb, gait and pace. Even its diet and behaviour transformed due to the changes in bone structure. This dramatization is a realistic and palpable one albeit being only fantasy.

Now it is not the purpose of this study to discuss this matter at any length but that this helps to illustrate these changes of a human's bones and their effects, and that may exemplify to a general audience the effect of bone remodelling over a period. Firstly, the different aspects of this essay will be individually discussed beginning with mechanical stress, followed by bone remodelling. Definitions will be brushed up and a walk through the cycle of bone remodelling and its fundamental constituents comes next. The different types of mechanical demands in activities of daily living will be sifted through and any misconceptions found fallible in literature are refined. The next part of the essay will focus on the association between the two and the effect that mechanical stress has on bone remodelling. Here, an account of stress fracture injuries is presented and the implications of mechanical stress on bone remodelling debated. The main factors affect bone remodelling through mechanical stress such as age, gender and ethnicity where possible. A brief overview of new medical applications in bone healing is outlined. Ultimately, the general conclusion from all this body of research is showed.

\section{Occupational Terminology}

Bone is a living rigid tissue that is subtly renewed throughout a person's life. The bones that make up the skeleton are not there just for structural support for the other bodily components but they serve a variety of functions. This includes acting as levers for the surrounding muscles to permit locomotion and movement in several planes; protecting vital internal organs from accidental damage; offering maintenance of intra- and extra-cellular levels of calcium and inorganic phosphate; serving as a stockpile of cytokines and growth factors; and providing the proper medium for hematopoiesis within the bone marrow. In all this, bone must be as lightweight as possible to decrease the overall body mass yet have the mechanical ability to endure stresses whenever a force is loaded upon them. This is mandatory for being able to carry out the conscious functions effortlessly, such as simply walking up the stairs.

\subsection{Mechanical Stress}

In physical terms, stress is the measure of the internal forces within a continuous material where particles standing adjacent to each other exert these forces on one other. Romani et al. concluded that "whether the stress comes from the pull of a muscle or the shock of a weight-bearing extremity contacting the ground, it is defined as the force applied per unit area of the load-bearing bone" [1]. There is also another type of stress, the physiological one. This can lead to the flight or fight response or if the state of stress prolongs over a longer period this response 
is stopped but the stress can have severe negative impact on a person's mental and physical well-being. Of course, there are also links between such psychological stress and diseases like osteoporosis that effect bone growth [2]. However, this is not the object of this paper and is to be distinguished from the more physical type of stress, that is mechanical stress that is the subject of the paper.

\subsection{Bone Remodelling}

The bone remodelling cycle preserves the integrity of the skeleton by strict control of two essentially opposing processes; bone resorption and bone formation, through the complementary toil of its constituent cell types. Bone resorption calls for the removal of mature bone from the skeleton and is accomplished by the bone-degrading osteoclast. The osteoclast cell has an exocrine function that dissolves the minerals in bone and then degrades extracellular matrix proteins by using enzymes. On the other hand, bone formation also known as ossification is as its name implies the growth of new bone tissue for which the bone-forming osteoblast is responsible for. The osteoblast cell produces the organic bone matrix and helps in its mineralization. Last but not least within bone matrix, the osteocyte acts as a mechanotransducer [3] and has a specific role in orchestrating osteoblastic and osteoclastic activity. A fourth cell type, "the bone lining cell, is thought to have a specific role in coupling bone resorption to bone formation, perhaps by physically defining bone remodelling compartments" [4].

\section{Mechanical Stress}

The quotidian natural forces experienced on bone are predominantly compression, tension, bending, torsion, and shear. Compression and tension are contrasting forces generated by attachments of ligament and muscle pulling on bone. Without cracking, compression causes bone to shorten and widen, thus reducing bone volume, while tension causes bone increase in volume as it lengthens and narrows. Miniscule levels of these forces cause bone to strain [1]. The mechanical aptitude of the bone's quality is reliant on material conformation of the bone's composite; geometry and architecture of the bone's network; and remodelling process repairing microdamage. These characteristics are influenced by external aspects like adaptation to the load itself and internal aspects like disease or aging [5].

The elasticity and strength of the bone to bear a load is determined by its structure and material composition. Trabecular bone exhibits elastic properties due to its intriguing morphological arrangement [5] but can withstand stress according to the alignment of fibre matrix [1]. The stiffness of bone correlates rather well to tissue mineralization according to compression and bending tests conducted on cortical bone [6] and in fact cortical bone can withstand stress in compression better than in tension [1].

Bone must be durable in a way that it is stiff to bear muscle forces or body weight, yet still able to resist deformation when the different types of mechanical 
stresses are applied on bone. Hence, throughout typical daily activity, bone must be able to shock absorb and deform accordingly when muscle forces are caused during nonimpact or impact exercises.

If the bone is made brittle in a sense that it is too stiff and unable to deform to an adequate degree, then the energy stored in the bone will be released and cause the structure to fail. Initially, microcracks will develop and eventually a full fracture will ensue. This may be observed in patients who suffer from osteogenesis imperfecta where the unfitting Type I collagen structure interacts differently with the hydroxyapatite crystals varying bone formation. In turn, collagen fibrils rapidly fail even at minor shearing forces [7]. On the other hand, if the bone is too flexible and deforms beyond peak strain thereby reaching the plastic range, it will also crack. Osteomalacia typically occurring in the elderly as it relates to factors like poor diet and being housebound lead to vitamin D deficiency. In basic terms, lack of vitamin D induces a fall in calcium and phosphate levels altering the mineralization process and deformed bowing on weight bearing bones is observed [8].

Despite this, Crockett et al. argue that the "presence of microcracks in bone affects mechanosensing and is currently considered a crucial driver of the remodelling response by initiating osteoclastic resorption" [4].

Acknowledging the influences required to maintain bone stability and the relationship with mechanical properties may lead to improvements in assessing a person's risk of fracture and in weighing the efficacy of new therapies and clinical tools such as prosthesis.

\section{Physiology of Bone Remodelling}

\subsection{Bone Modelling, Turnover and Remodelling}

Before heading onto the physiology of bone remodelling, I would like to point out the distinction made between modelling, turnover and remodelling which are often taken to mean the same. Modelling is the moulding of a malleable material into a particular shape. Therefore, bone being a biological material influenced by physical and physiological effects, continuously undergoes modelling to help it adapt to changing biomechanical forces [9]. On the other hand, remodelling removes old microdamaged bone and replaces it with new mechanically adaptive bone to help preserve the bone turnover rate [10].

Modelling is a process influenced by physiological stimuli where a gradual adjustment of the bone shape occurs due to the mechanical forces it encounters. The rate of bone growth is greater during childhood and adolescence as bone undergoes radial and longitudinal growth. Longitudinal growth occurs where cartilage proliferates in the epiphyseal and metaphyseal at the growth plates. It causes elongation of long bones, before subsequently undergoing mineralization to form primary new bone. Radial growth is the widening of bone or changing of axis by addition or removal of bone to the appropriate surfaces. The latter growth type does so, in response to endosteal resorption of old bone and perios- 
teal apposition of new bone, and normally keeps occurring with aging [9]. Throughout bone modelling, the formation and resorption pathways are not exactly matched. Bone modelling is less frequent than remodelling in adults.

In the case of bone turnover, measuring relevant bone biomarkers can provide an estimate on the total volume of bone. Thus, the term bone turnover is considered to be the normal replacement of bone tissue which occurs because of resorption and formation processes over a period of time that usually consists of approximately $10 \%$ of the skeleton every year [11]. Whereas, bone remodelling is the active process that maintains the microscopic change of bone structure throughout the coupled sequences of osteoclastic and osteoblastic activity. Hence, both terms are relatable in "cause and effect" respectively. This implies that the rate of bone remodelling refers to the dynamic physiological mechanism that influences bone turnover, while bone modelling does not participate directly to the physiological effects of bone turnover.

\subsection{The Constituents of Bone}

The skeleton of a new born baby is not fully ossified. At the edges of each long bone there is an epiphyseal plate that is an area of cartilage that diminishes with age until when maturity is reached it disappears almost entirely leaving only the epiphyseal line in adult life. Thus, by the end of adolescence, the adult weight of the skeleton is characteristically composed of $80 \%$ cortical bone and $20 \%$ trabecular cancellous bone. However, distinct types of bones and skeletal sites, including short, long, flat and irregular bones carry out distinct functions. In effect, due to their inherent function, within these different bones there are different ratios of cortical to trabecular bone found across the skeleton. For instance, the vertebra is composed in a ratio of $25: 75$, while femoral head is $50: 50$ and 95:5 in the radial diaphysis [9]. In addition, according to Romani et al., the outer shafts of long bones like the tibia are mainly composed of cortical bone, with a large percentage of trabecular bone towards the central portion of the shaft and the ends of the bone. While short and flat bones such as the tarsals and pelvis have a higher percentage of cancellous bone [1].

Cortical bone is highly packaged which makes it dense, strong and stiff. However, trabecular bone is more akin to a honeycomb meshwork of rods and plates interspersed in the bone marrow compartment. This provides a higher surface area for metabolic activity, for instance exchange of calcium. That is why, the more common interchangeable term for cortical bone is compact bone, and spongy bone is the common name for trabecular.

The fundamental unit of cortical bone is the osteon or Haversian system and that of trabecular bone is the trabeculae. The osteons consist of a central Haversian canal that house blood vessels and nerves. On the other hand, the trabeculae receive their neurovascular supply through canaliculi that are in turn connected to adjacent cavities, instead of a central Haversian canal. The matrix of osteons and trabeculae are arranged in concentric rings known as lamellae which resem- 
ble a rotated plywood structure. The way osteoblasts set down collagen fibrils in a lamellar pattern is yet to be discovered, although having a pattern in concentric fashion may provide substantial strength due to the alternating orientation of collagen fibrils [12]. In addition to trabeculae, the multiple bone packets are oriented parallel to the mutual line of stress but can realign their orientation of fibres if direction of stress alters accordingly [5]. Hence, as new bone is being formed, mechanical stress improves bone strength by influencing alignment of fibrils. Cortical bone tissue subjected to mostly tensile stresses has a higher percentage of collagen bras aligned along the long axis. On the other hand, in regions of predominant compressive stresses, bras are more likely to be aligned transverse to the bone long axis.

\subsection{Bone Remodelling Cycle}

Bone remodelling can be divided into the following five phases consecutively named; quiescent, activation, resorption, reversal, and formation. The quiescent phase is the state of inactivity of bone when at rest. The process of remodelling then begins with activation.

This stage involves a stimulus, predominantly a mechanical incentive and a detection mechanism towards the stimulus. Recent studies have shown that osteocytes are the primary sensing cells and the intensive connectivity makes them capable of picking up mechanical strain and relate the force of the strain with the appropriate transmission to regulate resorption and formation [3]. In fact, targeted deletion of osteocytes causes dysfunction to bone's response to mechanical loads resulting in bone loss [13]. However, investigations have shown that mechanical loading influences osteocytes to secrete transforming growth factor $\beta$ (TGF- $\beta$ ) [14], amongst others, resulting in osteoclastogenesis inhibition [15]. Disuse of limbs leading to immobilization or microdamage to the bone matrix made by the mechanical pulling of muscle and ligaments causes local osteocyte apoptosis [16]. In turn, focal levels of TGF- $\beta$ are reduced resulting in osteoclast activation. The recruitment of mononuclear monocyte-macrophage osteoclast precursors results in the fusion of multiple mononucleates to form large multinucleated osteoclasts [17]. Next in line is the resorption phase.

By referring to Figure 1 below, the mature osteoclasts fuse primarily to bone matrix using integrin receptors then secrete hydrogen $\left(\mathrm{H}^{+}\right)$ions and lysozymal enzymes principally cathepsin $\mathrm{K}$. A ruffled border is developed when $\mathrm{H}^{+}$ions acidify and dissolve the mineral component of the matrix. The low $\mathrm{pH}$ environment instils cathepsin $\mathrm{K}$ to digest the proteinaceous matrix since it is mostly made up of type I collagen. A resulting microscopic view reveals formation of Howship's lacunae that are irregular scalloped cavities on the trabecular bone surface, while cylindrical Haversian tunnels can be seen on cortical bone [10].

The reversal phase couples bone formation to bone resorption. In the final analysis, resorption cavities contain different cells including macrophages that finish the process of resorption and permit the release of the growth factors 


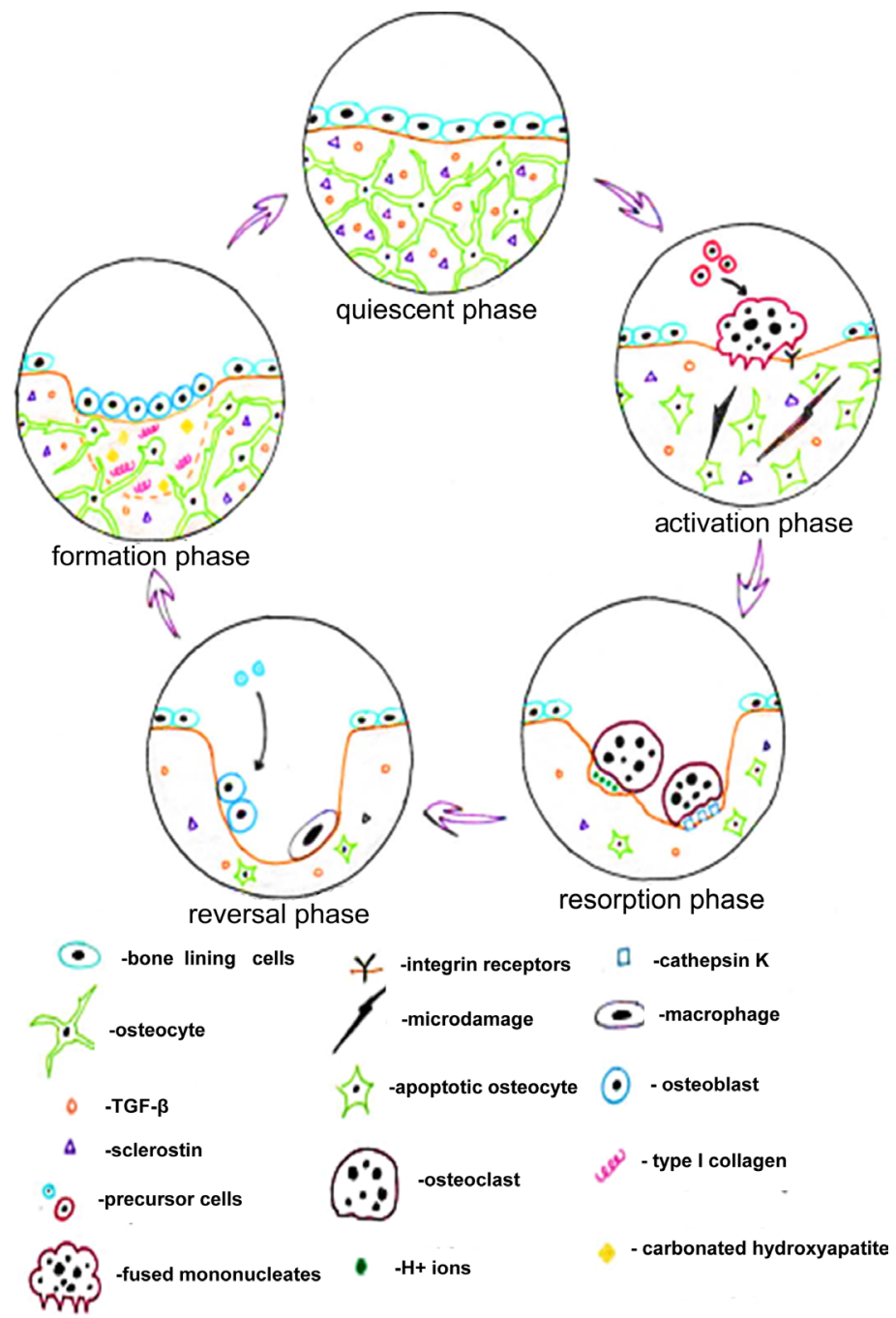

Figure 1. Bone remodelling cycle at cellular level.

contained within the matrix [18]. Preosteoblasts proliferate into osteoblasts that start new bone formation and osteocytes are freed from the newly digested bone matrix. The coupling signals linking the two processes are not yet fully known but they could include the bone morphogenetic proteins as they regulate early patterning of skeletal differentiation, and the Wingless signalling pathways (Wnt) particularly the Wnt/ $\beta$-catenin pathway that is vital for osteoblastic differentiation [4].

Once most of the osteoblastic lineage subpopulations of cells, including the mesenchymal stem cells and osteoprogenitor cells, have matured into mononucleated osteoblasts, the osteoclasts detach from the bone surface to allow the formation phase. On the resorption pits, osteoblasts synthesize and lay down the organic matrix ( $25 \%$ in volume) of $90 \%$ type 1 collagen and subsequently the mineral nanoparticles (70\% in volume) made of carbonated hydroxyapatite [5]. The individual osteoblasts are shown to be connected by adherens junctions and connected by gap junctions allowing cell-to-cell communication and hence work as an efficient unit. Some osteoblasts become trapped in their own newly made 
bone matrix. This gives rise to osteocytes which are confined within a space known as lacunae, and gradually stop secreting osteoid. And other osteoblasts, transform into quiescent bone lining cells that completely cover the newly formed bone surface [19]. These gap junctions eventually create a whole connecting system where the deeper layers of lamellae are linked with surface layers of lamellae through the onset network formation of canaliculi. Yellowley et al. injected a low molecular weight fluorescent dye into osteoblasts and showed that the dye diffused to surrounding and deeper cells in the osteon [20].

At this stage, the quiescent or "at rest" phase commences once more where the amount of bone resorbed should be equal to the amount of bone formed. The steady state of the bone surface is sustained until the next propagation of remodelling.

\section{Bone Remodelling and Mechanical Stress}

\subsection{A Stumbling Day at the Gym}

The XV Jeux des îles in Sicily were fast approaching in May 2011 and I was tediously practicing my flips on beam, tumbles on floor, rotations on bars and airtime on vault two months before the actual competition. Suddenly, it happened. It was March 12th when I was working on a new aerial skill on beam towards the end of my training session. I had been performing my beam routine over and over again for quite a long while but I just couldn't stick the landing of that new element. Even though I was the last gymnast still toiling away, I ignored my weariness and kept trying. Seeing that I wasn't getting much better from the previous nineteen tries, I decided to try it one last time before I called it a day.

I leapt in the air with all my might, rotated as quickly as I could, brought my arms in to balance out my body, but I landed uncomfortably on the edge of the beam. I heard a snap and a sudden rush of pain originating from my left foot. At the time, I didn't fuss about it nor did I alert my coach as I assumed the discomfort was something miniscule. I was sure that I would recover once I rested it. I limped as I walked out of the gym area but eventually I couldn't bear the pain so I hopped on my unaffected leg. I laboured up the 5-storey staircase slowly but surely. Once at the top, I waited for my father to arrive. As soon as he saw my situation, he rushed me to the Polyclinic to get it checked. An X-ray was taken and upon inspection, the third metatarsal exhibited a clear break and the fourth metatarsal appeared to be a hairline crack. The doctor quickly pointed out that this was a clear case of a stress fracture. In due course, I had to go to Mater Dei Hospital to get a cast done that had to last up to 6 weeks.

\subsection{Linking Bone Remodelling and Mechanical Stress}

The little scenario described above is from my own personal experience of the glory days in gymnastics. I picked this incident, from many, to explain and understand the implications of mechanical stress on bone remodelling with reference to stress fractures. 
Apart from other factors including: genetics, nutrition, vascular, neural and hormonal statuses; mechanical force plays a crucial role in bone remodelling in allowing bone to adapt its structure to the mechanical demands. These mechanical forces arise primarily from muscle actions that counteract external continuous impacting loads. This means that what bones experience changes them as Julian Wolff proposed at the end of the 19th Century. This is known as Wolff s law and was only refined more than half a century later by Harold Frost when he developed the Mechanostat model [21]. According to the Mechanostat, bones are capable of sensing mechanical loads and then they transform their architectural shape by fluctuations of bone remodelling. This aims to sustain bone competence to mechanical strain stimuli at an optimal level. Several mechanical thresholds control whether bone undergoes fluxes towards resorption or formation.

In lay terms, a weightlifter holding a $575 \mathrm{~kg}$ mass above him or even an experience of weightlessness can have stimulating or depressing effects on bone formation. According to Silva et al., baseball and tennis players show "geometric alterations favouring increased cortical thickness in the bone diaphysis" of the dominant humerus [22]. The muscular contraction transmits tension and compression to the bone, which is detected by the osteocyte network within the osseous tissue. The sloshing back and forth of the extracellular fluid is viscously enough to exert shear stress on the osteocyte membrane. This hypo-regulates osteocyte secretion of sclerostin, which consecutively allows Wnt signalling-dependent bone formation to occur [23] [24]. On the other hand, astronauts experiencing zero gravity in orbit illustrate profound bone loss since sclerostin becomes up regulated upon unloading periods which causes a turn off from osteoblastogenesis. Thus, to prevent substantial bone weakness, astronauts must undergo regular centrifugal forces that are equivalent with the earth's gravitational pull to sustain bone metabolism [25].

On looking at the mechanostat in detail, Figure 2 shows that remodelling rate happens in response to both loading and unloading with a difference in physiological occurrences. If the load exceeds point $\mathrm{B}$, indicating a state of loading beyond habituation, this causes an accumulation of microdamage. Thus, this increases bone remodelling particularly bone formation to strengthen the bone. On the other hand, below point $\mathrm{A}$, during a period of hoarded applications of unloading, bone remodelling also arises, however bone resorption is more pronounced [16]. A typical example of these influences on bone is seen in patients with paraplegia that are confined to a wheelchair. The lack of neural stimulation in the muscles of the paralytic legs decreases the functionality and activity of muscles hence negatively impacts bone to lose strength and mass as increasing rates of bone resorption and osteocyte apoptosis occur. However, the patient is still capable of using both arms to manoeuvre his whole-body weight around. This increases muscle mass and activity compared to the norm, thus, increasing bone mass and strength of the upper limbs [26]. 


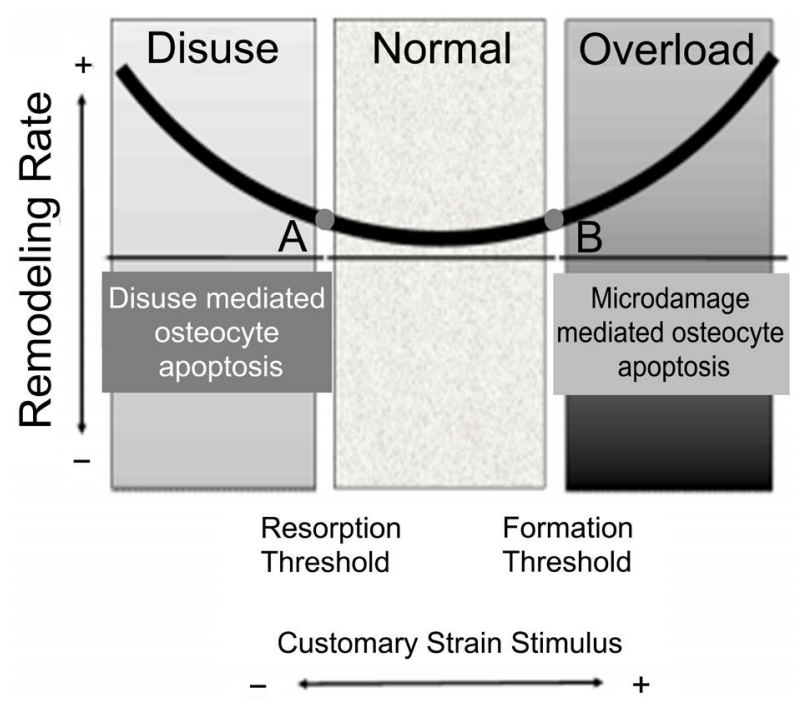

Figure 2. A graph shows a U-shaped manner remodelling rate in response to mechanical loading quantified in the customary strain stimulus. Point A reflects the resorption threshold and point B indicates the formation threshold [16].

\subsection{Cellular Manifestations}

Whole-animals have been sacrificed to vividly study the responses to mechanical stimuli at the tissue level and in vitro studies have confirmed that individual bone cells, such as osteoblasts and osteocytes, act as mechanoreceptors which help transduce the mechanical forces into biochemical signals [27]. Several investigations show performance of a non-invasive cantilever bending loading method with programmed wave forms for use on in vitro animals' tibia or ulna. These methods found that the mechanical strain induced was causing bone formation on the bone surface i.e. periosteum. The periosteum is assumed to be responsible for sensing mechanical strain possibly because the degree of stress experienced on a cylindrical volume is naturally found greatest on the surface and nearing to zero towards the centre.

Sakai et al. executed multiple experiments trying to identify if a cyclical loading, one of long-pulse and the other short-pulse stress modes, induced any cell proliferation or osteoblastic differentiation. The use of fluorescent imaging confirmed that the short pulse produced significant bone formation on the periosteal surface around the loading point; while in the long pulse, bone formation was momentous on the periosteal surface at the opposite side of the loading point. Mechanical loading seems to not only influence bone formation but also the whereabouts of formation itself that depends on the magnitude of the applied force. They also performed a progression of several histological analysis after the pulse mode was conducted. One test involved staining for alkaline phosphatase which is a ubiquitous plasma membrane bound enzyme but is sufficiently found in osteoblasts. A rise in alkaline phosphatase signals were observed at the hypertrophied periosteum indicating the strong presence of osteoblastic activity at this site. This further confirmed the findings of how me- 
chanical stress impacts remodelling and the repercussions caused on the bone's architecture. Indeed, through in-situ immunofluorescent imaging, periosteal cells were stained for detecting the act in cytoskeleton. The long pulse mode treatment indicated stronger signals of quick act in cytoskeletal disorganization soon after loading. This indicates the bone's immediate adaptability to load to resist a resultant fracture, hence increasing the malleability of bone's naturally rigid structure [28].

\subsection{A Walk through the Sequence of Events of the Incidental Stress Fracture}

A stress fracture is one of the commonest overuse injuries known to athletes. In a stress fracture, an onset single load will not fracture a bone but it is due to accumulated applications of submaximal stresses that exhaust the capacity of bone to remodel. Stress fractures are not exhibited as a full crack in the bone but may progress into one, as seen on the third metatarsal on X-ray image (Figure 3). Although, most typically only the bony cortex is involved in a stress fractures, in which case a hairline fracture may be visible there. Sports involving running or jumping such as track and field events, ballet, basketball, gymnastics, tennis carry the highest risk of tibia, fibula and metatarsal stress fractures. It is reported that more than $50 \%$ of stress fractures occur in the lower leg and foot as they bear most of the weight where a $0.7 \%$ to $20 \%$ are accounted for all sports injuries [29].

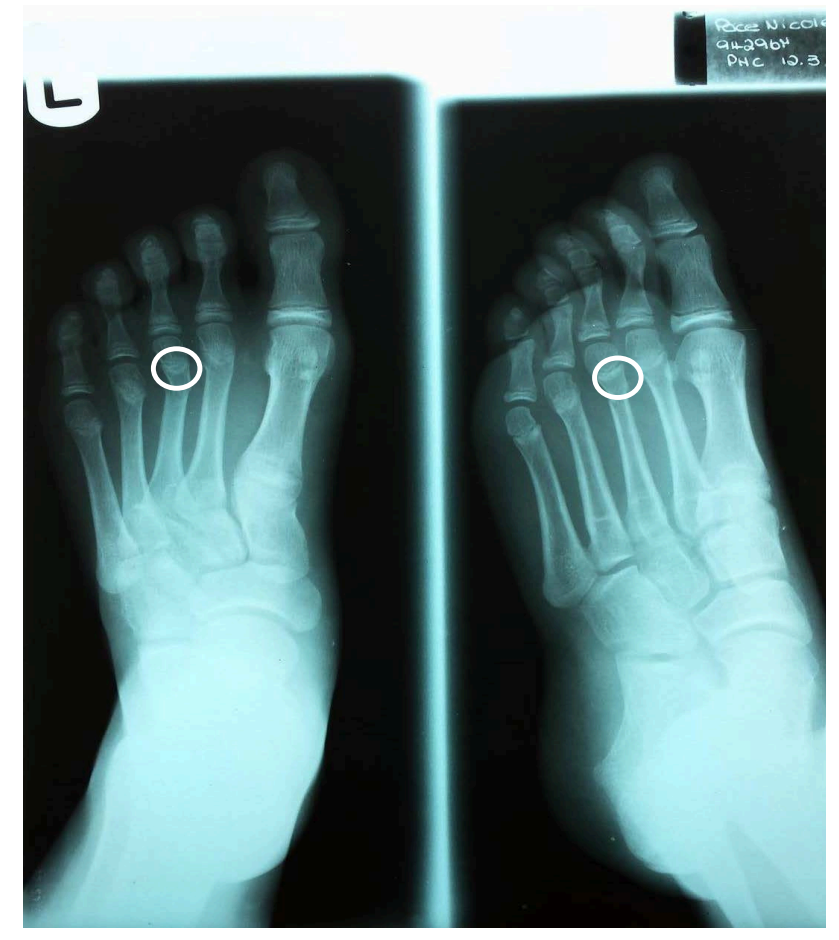

Figure 3. This is an X-ray of the left foot exhibiting the stress fractures. The white circle shows a clear break in the $3 \mathrm{rd}$ metatarsal. The hairline crack in the 4th metatarsal is not clearly visible above but showed on a later X-ray that is not available here. 
The commonest cause in the physically active population is fatigue. The events that led to my injury and caused the deterioration of healthy bones may result from the combined effects of increased training intensity; hard training surface of the beam; slightly nutrition deficient towards end of training; and poor anatomical alignment of feet. It is implied that the monotonous loading causes a brief moment of blood flow cut off and restricted oxygen perfusion to the area of bone stressed [1]. This restriction signals osteocytes to channel into the bone causing an increase in remodelling. The generated remodelling cannot compensate over the induced strain resulting in onset weakness of bone that is less able to withstand subsequent loads and hence the stress fracture.

The management ensuing the stress fracture requires immediate rest to take advantage of the physiological healing process. This allows time for proper maturation of the osteoblastic and monocyte macrophage lineages; healing of blood vessels to prevent ischemic injury; and development of periosteum. Prior to the formation of lamellar bone, woven bone is made as osteoblasts produce osteoid hastily by laying down collagen fibrils in a haphazard organization. Subsequent bone remodelling occurs where the deposition of resilient lamellar bone is formed [10].

Casting at injured site allows a protected and immobilized environment. But a patient that presents with a stress fracture may avoid casting the affected area especially a physically active person. This is because the casting depresses muscle stimulation as they are not being put to use over a substantial amount of time thus forces on bone's surface are constantly being reduced and subsequently bone will start to atrophy and weaken. Therefore, it is recommended to use crutches and the foot dangles so that minor ligament and muscle pulls can still act on bone. However, in my case a plaster cast was still considered due to constant displacement from one room to another through the school and reduced the risk of fracture once again upon incidental falling or uneasy stepping. The following 3 to 4 weeks after cast was removed a period of rehabilitation was required. Upon which the atrophied leg muscles were stimulated and non-stressful weight bearing exercises where performed until acute symptoms no longer occurred during daily activities.

\subsection{The Variables of Mechanical Stress on Bone Remodelling}

To summarize, the force and/or loading type, magnitude, duration and number of repetitions of a mechanical stress can have drastic effects on bone remodelling. The different types of natural forces (including compression, tension, traction, torsion, bending, shear, torque) of mechanical deformations are not clear how differently they affect bone remodelling. Nevertheless, these forces can be quantified in strain that is the change in length relative to the original length and is accepted to induce mechanotransduction [25]. Secondly, according to Frost, there is a positive correlation between muscle and bone cross sectional areas [21]. This implies that the magnitude of the mechanical loading is proportional 
to the intensity of the bone remodelling. The bigger the compressive and tensile forces of the muscles and ligaments acting on bone or the bigger the strain exhibited on bone as it shock-absorbs an impact; the greater the impression on osteogenesis.

Moreover, adverse effects on bone remodelling are caused due to the loading types that fundamentally are dynamic and static loading. Static loading is a force that is relentless for an extended amount of time and causes only marginal inducement to remodelling. On the other hand, dynamic loading is a force applied rapidly over time that causes a more prompt and profound stimulus on remodelling. An average person that participates in regular activity promotes bone strength through proper perfusion of nutrients to osteocytes across the many layers bone and a standard bone remodelling is maintained [1]. However, to optimize bone quality resting periods are recommended between loading phases. The resting stage should still be quite active by limiting the extraordinary stressful loads squished in short period of time and by preventing the progression of a sedentary lifestyle. If the muscle activity suddenly diminishes over a lengthy period of time, the forces acting on bone's surface are decreased correspondingly and may drive away from osteoblastogenesis and into adipogenesis [9].

This cyclical pattern of loading and unloading can prevent desensitization of the mechanotransduction ability of the bone resulting in a "greater than 100-fold increase in fatigue resistance, despite a much smaller absolute gain in bone mass" [24]. The cycle of training develops the skeleton for further loads by producing micro stress fractures on the bones that grow stronger once remodelled. In this way further training produces more micro cracks and ultimately stronger bones [30].

\section{Other Factors Affecting Bone Together with Mechanical Stress}

\subsection{Age}

In the womb, the formation of foetal bone undergoes intramembranous ossification that primarily creates woven bone and through subsequent differentiation of osteogenic cells and angiogenesis, a more robust lamellar bone is formed [31]. But it does not end there. Bones must lengthen, widen and strengthen as one needs to grow to reach a potential height and weight to meet daily work activities more effectively.

The large amount of bone mineral stores is predominantly found across the physical growth phase between childhood and adolescence. The high mineralization rate and growth spurt are visible right up to sexual maturity. The multipart interaction of nutrients, genes, hormones and mechanical demands cause bone cells to proliferate at the growth plate of long bones. Towards the end of puberty, increase production of sex steroids particularly oestrogen from both the reproductive glands in males and females causes the growth plates of bones to fuse. This fusion closes the growth centres of long bones and renders them una- 
ble to respond effectively to the hormones that initiate growth.

As the reduction of bone turnover in adulthood takes its toll, a decrease in the levels of oestrogen results in an increase rate of bone resorption [32]. The overall effect is that the porous spaces between the trabecular network increase in size and the "presence of empty lacunae in aging bone suggests that osteocytes may undergo apoptosis" [10]. This plays a major role in the development of osteoporosis and related fractures especially in postmenopausal women. Literature implies that oestrogen and bisphosphonate therapy of bone may help prevent osteoblast and osteocyte apoptosis and hence reduce the chances of developing osteoporosis [33] [34].

\subsection{Gender}

Periosteal apposition in aging is considered advantageous as the bone's cross-sectional area increases and a rise in bone marrow space [35] indicates improvement of medullary hematopoiesis and beneficial effects on spleen and thymus [36]. Despite this, endocortical resorption increases correspondingly to this change. If endocortical resorption exceeds periosteal apposition then a net increase in cortical porosity and a large decrease in trabecular bone mineral density (BMD) succeed.

In relation to bone remodelling differences between the genders, in young adulthood, men in consistency with their averagely larger body size have 35\% $42 \%$ larger bone areas than women [37]. At central sites in both sexes, bone area is increased equally over life while at peripheral sites, men gain slightly more bone area [38]. The higher levels of testosterone in males contribute to an effective gain in muscle bulk upon physical activity, hence correspondingly stimulating periosteal growth [39]. The deceleration in limb growth and acceleration in trunk growth are partly oestrogen dependent [40]. This agrees with the typical development of longer legs as males' pubertal growth spurt reaches a higher and longer peak velocity, then epiphyseal fusion occurs at a later stage.

In athletes, it is theoretically recognised that females have a higher incidence of injury. The higher oestrogen levels, more body fat stored and greater flexibility of joints parallels along with less muscle mass and less powerful muscles. These factors less effectively stimulate bone remodelling when comparing performance of the same sporting activity from both genders. In addition, a blend of irregular menstrual periods, inadequate nutrient intake, and overall bone loss contribute to what is known as the "female athlete triad". In fact, the incidence of stress fractures in female athletes is $3.2 \%$ more than the males [41]. Hence, athletes with a stress fracture or inclining towards one particularly in females should be examined by taking a detailed medical history and in females possibly identify a presence of the triad to help prevent setbacks in the practice of sport.

\subsection{Ethnicity}

A disparity also exists among the races in both sexes. Investigations have shown 
that blacks have the highest overall BMD compared to whites, Asians and Hispanics, men and women. Longitudinal growth of the femur is found to be most rapid in blacks and the least in Asians, while longitudinal vertebral growth is found vice versa with Asians having the quickest growth and slowest in blacks. To put it in perspective, blacks have longer legs and shorter trunks than whites, Asians have shorter legs and longer trunks than whites and Hispanics are overall shorter than whites but they have similar body proportions as whites [42].

Therefore, throughout life, blacks seem to have the highest bone strength. The several factors contributing to the development of a stronger skeleton during childhood and adolescence that impact blacks' distinctive bone strength is dependent on the interaction of genetic and environmental factors. This possibly includes nutritional dependencies where during times of relative calcium deficiency, conservation of calcium from non-skeletal sources (most likely renal) is found greater than in whites. They have also acquired decreased skeletal sensitivity to acute resorptive effects [43].

In adulthood, blacks have slower bone loss processes due to the reduced rates of bone turnover and greater capacity of bone formation due to the incremental improvement of calcium homeostasis. This overall increase in bone mass in blacks correspond to the lowest incidence of osteoporotic fractures in black women, and in the military, blacks and Hispanic men and women have shown a lower incidence of stress fractures than whites. Regarding Asians, it is speculated that long bone epiphyses and vertebral growth are greatly more sensitive to oestrogen than whites, Hispanics and blacks, but this clearly needs further investigating [42].

\section{Innovative Treatments and Novel Diagnoses}

New ways of treating and diagnosing common bone disorders have emerged as a result of all this study into bone remodelling and mechanical stress. This is particularly due to better conception of common genetic variants and dissimilarities in bone remodelling across these variants; in the use of novel drugs in medicine and in daily loading and healing of bones.

" $C B D$ leads to improvement in fracture healing and demonstrate the critical mechanical role of collagen crosslinking enzymes." [44].

A recent study made on rat mid femoral shaft fractures has shown that cannabinoid (CBD), a non-psychoactive cannabis component can effectively speed up the healing process and further strengthen the bone after incidental fracture. Without the unnecessary side effects, the CBD receptors located throughout the skeletal system can prevent bone loss as they stimulate bone formation. In future, this could be a full proof therapeutic method in the aging population where the risk of osteoporosis and fragility fractures is more prominent [ibid].

"The basis of treatment of the individual patient with osteoporosis in the future, as it occurs in other chronic conditions" [45].

This study focused on the genetics and pathophysiology of rare skeletal dis- 
eases that can expand our understanding of the actual effectiveness of new treatments for osteoporosis on bone metabolism [ibid].

"Are SCs (stem cells) ... the daddy of all cure and medical interventions?" [46].

The bone-forming osteoblasts originate from mesenchymal stem cells (MSC). MSCs can also proliferate into chondrocytes capable of producing cartilage that will act as a "template for additional bone formation through the process of endochondral ossification" [47]. Therapeutic application of MSCs has significant impact in increasing the recovery rate of a fracture and aid in cartilage repair against the chondro-degenerative pathogenesis of osteoarthritis (OA). However, the elderly and/or obese individuals that contribute to most of the OA cases show decreased differentiation potential [46].

"Normal walking produces strains inside the crack of an incomplete atypical fracture that greatly exceed the limit for bone formation." [48].

Studies in atypical femoral fractures are showing that bone formation is not sufficient to heal the fracture. Using clinical CT and high resolution $\mu \mathrm{CT}$ images, patient-specific model explains why these fractures were not healing properly. The models predicted large strain distributions produced just by normal walking that the body could not cope with in order to heal. Bone would only form in less than $1 \%-5 \%$ of the crack volume which implies impaired healing usually witnessed in incomplete atypical fractures. A realistic microgeometry needs to be actualized when programming healing since the crack itself was shown to be healing only in parts and necrotic material was copiously contained and what's worse is that it seemed to have been enlarged by resorption [ibid].

These are but three areas that are highlighted above and serve but as an example of how research into medical theory can support and point out improved medical treatments offered to patients.

\section{Conclusion}

Bone remodelling is a rather complex that is affected by a multitude of variant factors, of which mechanical stress is one of them yet the association it has with bone remodelling is not fully understood. However, in this essay I have tried to analyse the various components of this multifaceted issue and to dissect into the different mechanisms of bone remodelling and how different types of mechanical stress contribute to their work on the bones. Understanding the interplay of mechanical stress that drives bone remodelling to create the desired bone strength would make medical practice better and ultimately the lives of patients more liveable. In the prepubertal years, exercise enhanced by dynamic cyclical load with rest periods may promote periosteal gains and high bone strength. Daily mechanical needs should be maintained later in life to sustain bone mass and thus slow down bone deterioration, reduce falls and associated fragility fractures and overall improve quality of life. This may be just forlorn, but I believe it is achievable with the intervention of proper medical techniques. Hope it is a change for the better: one from Gollum to Sméagol rather than vice versa. 


\section{Acknowledgements}

My sincerest gratitude goes to Dr Simon Camilleri for being my tutor and for giving me support in this field. I greatly appreciate his genuine effort for providing me with constructive guidance throughout this project. Best thanks must definitely go to my thoughtful family. My sisters Raquel and Gyllain and my brother Pierre who have been a wonderful source for a good laugh and that turned my dimmest of times into joyful ones. My loving parents, Jacqueline and Edmund Pace for their unceasing inspiration and for their affectionate support through the foggiest of fogs. They have taught me in so many ways and on so many aspects through the teachings of the bible. Above all I am very grateful for God and His unconditional love despite my limitations and many detours.

\section{References}

[1] Romani, W.A., Gieck, J.H., Perrin, D.H., Saliba, E.N. and Kahler, D.M. (2002) Mechanisms and Management of Stress Fractures in Physically Active Persons. Journal of Athletic Training, 37, 306-314.

[2] Kumano, H. (2005) Osteoporosis and Stress. Clinical Calcium, 15, 1544-1547.

[3] Bonewald, L.F. and Johnson, M.L. (2008) Osteocytes, Mechanosensing and Wnt Signalling. Bone, 42, 606-615. https://doi.org/10.1016/j.bone.2007.12.224

[4] Crockett, J.C., Rogers, M.J., Coxon, F.P., Hocking, L.J. and Helfrich, M.H. (2011) Bone Remodelling at a Glance. Journal of Cell Science, 124, 991-998. https://doi.org/10.1242/jcs.063032

[5] Carretta, R., Lorenzetti, S. and Müller, R. (2013) Towards Patient-Specific Material Modeling of Trabecular Bone Post-Yield Behaviour. The International Journal for Numerical Methods in Biomedical Engineering, 29, 250-272. https://doi.org/10.1002/cnm.2516

[6] Keller, T.S. (1994) Predicting the Compressive Mechanical Behaviour of Bone. Journal of Biomechanics, 27, 1159-1168. https://doi.org/10.1016/0021-9290(94)90056-6

[7] Gautieri, A., Uzel, S., Vesentini, S., Redaelli, A. and Buehler, M.J. (2009) Molecular and Mesoscale Mechanisms of Osteogenesis Imperfecta Disease in Collagen Fibrils. Biophysical Journal, 97, 857-865. https://doi.org/10.1016/j.bpj.2009.04.059

[8] Rokan, Z. and Kealey, W.D. (2015) Osteomalacia: A Forgotten Cause of Fractures in the Elderly. BMJ Case Reports, 2015, bcr2014207184.

https://doi.org/10.1136/bcr-2014-207184

[9] Clarke, B. (2008) Normal Bone Anatomy and Physiology. Clinical Journal of the American Society of Nephrology: CJASN, 3, S131-S139. https://doi.org/10.2215/CJN.04151206

[10] Kini, U. and Nandeesh, B.N. (2012) Physiology of Bone Formation, Remodelling, and Metabolism. In: Fogelman, I., Gnanasegaran, G. and Wall, H., Eds., Radionuclide and Hybrid Bone Imaging, Springer, Berlin, Heidelberg, 29-57.

[11] Parfitt, A.M. (2004) What Is the Normal Rate of Bone Remodelling? Bone, 35, 1-3. https://doi.org/10.1016/j.bone.2004.03.022

[12] Yamamoto, T., Hasegawa, T., Sasaki, M., Hongo, H., Tabata, C., Liu, Z., Li, M. and Amizuka, N. (2012) Structure and Formation of the Twisted Plywood Pattern of Collagen Fibrils in Rat Lamellar Bone. Journal of Electron Microscopy, 61, 113-121. 
https://doi.org/10.1093/jmicro/dfs033

[13] Tatsumi, S., Ishii, K., Amizuka, N., Li, M., Kobayashi, T., Kohno, K., Ito, M., Takeshita, S. and Ikeda, K. (2007) Targeted Ablation of Osteocytes Induces Osteoporosis with Defective Mechanotransduction. Cell Metabolism, 5, 464-475. https://doi.org/10.1016/j.cmet.2007.05.001

[14] Raggatt, L.J. and Partridge, N.C. (2010) Cellular and Molecular Mechanisms of Bone Remodelling. Journal of Biological Chemistry, 285, 25103-25108. https://doi.org/10.1074/jbc.R109.041087

[15] You, L., Temiyasathit, S., Lee, P., Kim, C.H., Tummala, P., Yao, W., Kingery, W., Malone, A.M., Kwon, R.Y. and Jacobs, C.R. (2007) Osteocytes as Mechanosensors in the Inhibition of Bone Resorption Due to Mechanical Loading. Bone, 42, 172-179. https://doi.org/10.1016/j.bone.2007.09.047

[16] Hughes, J.M. (2011) Revisiting Harold Frost's Mechanostat Theory of Bone Functional Adaptation: New Interpretations Based on New Evidence.

[17] Bruzzaniti, A. and Baron, R. (2006) Molecular Regulation of Osteoclast Activity. Reviews in Endocrine and Metabolic Disorders, 7, 123-139. https://doi.org/10.1007/s11154-006-9009-x

[18] Sims, N.A. and Martin, T. (2014) Coupling the Activities of Bone Formation and Resorption: A Multitude of Signals within the Basic Multi-Cellular Unit. BoneKEy Reports, 3, Article No. 481.

[19] Ruimerman, R., Rietbergen, B., Hilbers, P. and Huiskes, R. (2005) The Effects of Trabecular-Bone Loading Variables on the Surface Signaling Potential for Bone Remodelling and Adaptation. Annals of Biomedical Engineering, 33, 71-78. https://doi.org/10.1007/s10439-005-8964-9

[20] Yellowley, C.E., Li, Z., Zhou, Z., Jacobs, C.R. and Donahue, H.J. (2000) Functional Gap Junctions between Osteocytic and Osteoblastic Cells. Journal of Bone and Mineral Research, 15, 209-217. https://doi.org/10.1359/jbmr.2000.15.2.209

[21] Frost, H.M. (1997) Defining Osteopenias and Osteoporoses: Another View (with Insights from a New Paradigm). Bone, 20, 385-391. https://doi.org/10.1016/S8756-3282(97)00019-7

[22] Silva, C., Goldberg, T., Teixeira, A. and Dalmas, J. (2011) The Impact of Different Types of Physical Activity on Total and Regional Bone Mineral Density in Young Brazilian Athletes. Journal of Sports Sciences, 29, 227-234.

[23] Turner, C.H., Warden, S.J., Bellido, T., Plotkin, L.I., Kumar, N., Jasiuk, I., Danzig, J. and Robling, A.G. (2009) Mechanobiology of the Skeleton. Science Signalling, 2, pt3. https://doi.org/10.1126/scisignal.268pt3

[24] Schwab, P. and Scalapino, K. (2011) Exercise for Bone Health: Rationale and Prescription. Current Opinion in Rheumatology, 23, 137-141.

[25] Nagaraja, P.M. and Jo, H. (2014) The Role of Mechanical Stimulation in Recovery of Bone Loss-High versus Low Magnitude and Frequency of Force. Life, 4, 117-130. https://doi.org/10.3390/life4020117

[26] Dionyssiotis, Y., Stathopoulos, K., Trovas, G., Papaioannou, N., Skarantavos, G. and Papagelopoulos, P. (2015) Impact on Bone and Muscle Area after Spinal Cord Injury. BoneKEy Reports, 4, 633. https://doi.org/10.1038/bonekey.2014.128

[27] Marie, P.J. (2011) Osteoblast Biology and Mechanosensing. In: Noda, M., Ed., Mechanosensing Biology, Springer Japan, Tokyo, 105-126. https://doi.org/10.1007/978-4-431-89757-6_8

[28] Sakai, D., Kii, I., Nakagawa, K., Matsumoto, H.N., Takahashi, M., Yoshida, S., Ho- 
soya, T., Takakuda, K. and Kudo, A. (2011) Remodelling of Actin Cytoskeleton in Mouse Periosteal Cells under Mechanical Loading Induces Periosteal Cell Proliferation during Bone Formation. PLoS ONE, 6, e24847.

https://doi.org/10.1371/journal.pone.0024847

[29] Snyder, R.A., Koester, M.C. and Dunn, W.R. (2006) Epidemiology of Stress Fractures. Clinics in Sports Medicine, 25, 37-52.

[30] Andreoli, A., Celi, M., Volpe, S.L., Sorge, R. and Tarantino, U. (2012) Long-Term Effect of Exercise on Bone Mineral Density and Body Composition in Post-Menopausal Ex-Elite Athletes: A Retrospective Study. European Journal of Clinical Nutrition, 66, 69-74. https://doi.org/10.1038/ejcn.2011.104

[31] Dimitriou, R., Jones, E., McGonagle, D. and Giannoudis, P.V. (2011) Bone Regeneration: Current Concepts and Future Directions. BMC Medicine, 9, 66.

https://doi.org/10.1186/1741-7015-9-66

[32] Krassas, G. and Papadopoulou, P. (2001) Oestrogen Action on Bone Cells. Journal of Musculoskeletal and Neuronal Interactions, 2, 143-151.

[33] Fadanelli, M. and Bone, H. (2004) Combining Bisphosphonates with Hormone Therapy for Postmenopausal Osteoporosis. Treatments in Endocrinology, 3, 361-369. https://doi.org/10.2165/00024677-200403060-00004

[34] Adler, R.A., El-Hajj Fuleihan, G., Bauer, D.C., Camacho, P.M., Clarke, B.L., Clines, G.A., Compston, J.E., Drake, M.T., Edwards, B.J., Favus, M.J., Greenspan, S.L., McKinney, R., Pignolo, R.J. and Sellmeyer, D.E. (2016) Managing Osteoporosis in Patients on Long-Term Bisphosphonate Treatment: Report of a Task Force of the American Society for Bone and Mineral Research. Journal of Bone and Mineral Research, 31, 16-35. https://doi.org/10.1002/jbmr.2708

[35] Jepsen, K.J. and Andarawis-Puri, N. (2012) The Amount of Periosteal Apposition Required to Maintain Bone Strength during Aging Depends on Adult Bone Morphology and Tissue-Modulus Degradation Rate. Journal of Bone and Mineral Research, 27, 1916-1926. https://doi.org/10.1002/jbmr.1643

[36] Lo Iacono, N., Blair, H.C., Poliani, P.L., Marrella, V., Ficara, F., Cassani, B., Facchetti, F., Fontana, E., Guerrini, M.M., Traggiai, E., Schena, F., Paulis, M., Mantero, S., Inforzato, A., Valaperta, S., Pangrazio, A., et al. (2012) Osteopetrosis Rescue upon RANKL Administration to Rankl (-/-) Mice: A New Therapy for Human RANKL-Dependent ARO. Journal of Bone and Mineral Research, 27, 2501-2510. https://doi.org/10.1002/jbmr.1712

[37] Riggs, B.L., Melton, L.J., Robb, R.A., Camp, J.J., Atkinson, E.J., Peterson, J.M., Rouleau, P.A., McCollough, C.H., Bouxsein, M.L. and Khosla, S. (2004) Population-Based Study of Age and Sex Differences in Bone Volumetric Density, Size, Geometry, and Structure at Different Skeletal Sites. Journal of Bone and Mineral Research, 19, 1945-1954. https://doi.org/10.1359/jbmr.040916

[38] Farr, J.N. and Khosla, S. (2015) Skeletal Changes through the Lifespan Mdash from Growth to Senescence. Nature Reviews Endocrinology, 11, 513-521. https://doi.org/10.1038/nrendo.2015.89

[39] Brown, M. (2008) Skeletal Muscle and Bone: Effect of Sex Steroids and Aging. Advances in Physiology Education, 32, 120-126. https://doi.org/10.1152/advan.90111.2008

[40] Singh, D., Sanyal, S. and Chattopadhyay, N. (2010) The Role of Estrogen in Bone Growth and Formation: Changes at Puberty. Cell Health and Cytoskeleton, 3, 1-12.

[41] Chen, Y., Tenforde, A.S. and Fredericson, M. (2013) Update on Stress Fractures in Female Athletes: Epidemiology, Treatment, and Prevention. Current Reviews in 
Musculoskeletal Medicine, 6, 173-181. https://doi.org/10.1007/s12178-013-9167-x

[42] Seeman, E. (1998) Growth in Bone Mass and Size-Are Racial and Gender Differences in Bone Mineral Density More Apparent than Real? The Journal of Clinical Endocrinology and Metabolism, 83, 1414-1419.

[43] Cosman, F., Morgan, D.C., Nieves, J.W., Shen, V., Luckey, M.M., Dempster, D.W., Lindsay, R. and Parisien, M. (1997) Resistance to Bone Resorbing Effects of PTH in Black Women. Journal of Bone and Mineral Research, 12, 958-966. https://doi.org/10.1359/jbmr.1997.12.6.958

[44] Kogan, N.M., Melamed, E., Wasserman, E., Raphael, B., Breuer, A., Stok, K.S., Sondergaard, R., Escudero, A.V., Baraghithy, S., Attar-Namdar, M., Friedlander-Barenboim, S., Mathavan, N., Isaksson, H., Mechoulam, R., Müller, R., Bajayo, A., et al. (2015) Cannabidiol, a Major Non-Psychotropic Cannabis Constituent Enhances Fracture Healing and Stimulates Lysyl Hydroxylase Activity in Osteoblasts. Journal of Bone and Mineral Research, 30, 1905-1913.

https://doi.org/10.1002/jbmr.2513

[45] Appleman-Dijkstra, N.M. and Papapoulos, S.E. (2016) From Disease to Treatment: From Rare Skeletal Disorders to Treatments for Osteoporosis. Endocrine, 52, 414-426. https://doi.org/10.1007/s12020-016-0888-7

[46] Pace, P. and Blundell, R. (2016) Stem Cells: Daddy or Chips?-An Up-to-Date Review on Ground-Breaking Discoveries in Stem Cell Research, with Special Attention to iPSC Applications in Osteoarthritis. Stem Cell Discovery, 6, 39-48. https://doi.org/10.4236/scd.2016.61004

[47] Knight, M.N. and Hankenson, K.D. (2013) Mesenchymal Stem Cells in Bone Regeneration. Advances in Wound Care, 2, 306-316. https://doi.org/10.1089/wound.2012.0420

[48] Gustafsson, A., Schilcher, J., Grassi, L., Aspenberg, P. and Isaksson, H. (2016) Strains Caused by Daily Loading Might Be Responsible for Delayed Healing of an Incomplete Atypical Femoral Fracture. Bone, 88, 125-130.

https://doi.org/10.1016/j.bone.2016.04.020 